\title{
Decreased Lempel-Ziv complexity in Alzheimer's disease patients' magnetoencephalograms
}

\author{
Carlos Gómez*, Roberto Hornero*, Alberto Fernández ${ }^{\dagger}$, Daniel Abásolo* and Miguel López* \\ *E.T.S. Ingenieros de Telecomunicación, Universidad de Valladolid, Spain. Email: cgompen@ ribera.tel.uva.es \\ ${ }^{\dagger}$ Centro de Magnetoencefalografía Dr. Pérez-Modrego, Universidad Complutense de Madrid, Spain
}

\begin{abstract}
The aim of the present research is to study the magnetoencephalogram (MEG) background activity in patients with Alzheimer's disease (AD) using the Lempel-Ziv (LZ) complexity. We recorded the MEG with a 148-channel wholehead magnetometer (MAGNES 2500 WH, 4D Neuroimaging) in 10 patients with probable $\mathrm{AD}$ and 10 age-matched control subjects, during five minutes. Artefact-free epochs were selected for the non-linear analysis. In all MEG channels, the AD patients had lower complexity than control subjects. In 77 of them the differences were statistically significant $(p<0.01)$. These preliminary results suggest that cognitive dysfunction in AD is associated with a decreased complexity in certain regions of the brain.
\end{abstract}

\section{INTRODUCTION}

Alzheimer's disease (AD) is one of the most frequent disorders among the elderly population [1], [2] and it is considered to be the main cause of dementia in western countries [3]. This degenerative neurological disease is characterized by neuronal loss and the appearance of neuritic plaques containing amyloid- $\beta$-peptide and neurofibrillary tangles [4], [5]. The differential diagnosis with other types of dementia includes Mini-Mental Status Examination (MMSE) of Folstein [6], Stroop test, computerized tomography and magnetic resonance imaging, although a definitive diagnosis is only possible by necropsy.

The exact nature of the neurophysiological processes underlying cognitive dysfunction in $\mathrm{AD}$ is still incompletely understood [7]. There are several studies of the electroencephalogram (EEG) and magnetoencephalogram (MEG) in $\mathrm{AD}$ patients with non-linear methods. The most widely used method is the correlation dimension $\left(D_{2}\right)$ [8], [9]. Nevertheless, there are several drawbacks in using this measure for estimating the non-linear dynamic complexity. First, the algorithm requires the data series to be stationary [10], which cannot be achieved for physiological data. In addition, it is necessary long time series to obtain meaningful results [11]. Moreover, the reconstruction of the attractor in the phase space is computationally very expensive. In spite of its shortcomings, recently, $D_{2}$ was computed and compared with another measure of neural complexity $\left(C_{N}\right)$ in $\mathrm{AD}$ patients' MEGs [12]. Other methods also have been used. For instance, Stam et al. [7] detected that the mean level of EEG synchronization was lower in $\mathrm{AD}$ patients in the upper alpha (10-13 Hz) and beta (13-30 Hz) bands, and that the spontaneous fluctuations of synchronization diminished in $\mathrm{AD}$ patients in the lower alpha $(8-10 \mathrm{~Hz})$ and beta (13-30
$\mathrm{Hz}$ ) bands. Other studies found that the local cross-mutual information [13] and the Global Field Synchronization [14] can distinguish between control subjects and AD patients.

The LZ complexity [15] is a nonparametric measure of complexity in a one-dimensional signal related to the number of distinct substrings and the rate of their recurrence. Huang et al. [16] applied this method to characterize the functional changes of the brain during isoflurane anaesthesia, extracting the complexity from mutual information time series of EEGs. Moreover, preliminary evidence suggests that, applied to EEGs, LZ complexity is very sensitive to the extent of focal ischemic cerebral injury, being able to distinguish ischemic and normal region [17]. It has been applied to study the brain function [18], brain information transmission [19] and to detect ventricular tachycardia and fibrillation [20]. It can also be used for quantifying the regularity at epochs of epileptic seizures time-series data [21], and the relationship between brain activity patterns and depth of anesthesia [22], [23].

In the present study, we have examined the MEG background activity in $\mathrm{AD}$ patients with the LZ complexity. The purpose of this research is to test the hypothesis that the LZ complexity measure applied to MEG data is lower in AD patients compared to control subjects.

\section{Methods AND MATERIALS}

\section{A. Subjects}

Ten patients $($ age $=66.3 \pm 7.1$ years, mean \pm standard deviation SD) fulfilling the criteria of probable $\mathrm{AD}$ and ten control subjects (mean age, $65.8 \pm 5.9$ SD years) participated in this study. Participants were recruited from the Asociación de Familiares de Enfermos de Alzheimer (AFAL). All patients with $\mathrm{AD}$ were diagnosed using criteria of the National Institute of Communicative Disorders and Stroke and the AD and Related Disorders Association (NINCDS-ADRDA) [24]. The mean MMSE score for the patients was $15.5 \pm 4.1$ (Mean \pm SD). Two of them had a MMSE score of less than 12 points, indicating a severe degree of dementia. None of the patients used any kind of medication that could have an influence on the MEG. For the control subjects the MMSE score was $29.4 \pm 1.0$ (Mean $\pm \mathrm{SD}$ ). The main characteristics of all subjects are summarized in table I.

The local ethics committee approved the study. All control subjects and all caregivers of the demented patients gave their informed consent for the participation in the current study. 
TABLE I

SOCIODEMOGRAPHIC DATA OF CONTROLS SUBJECTS AND PATIENTS WITH ALZHEIMER DISEASE

\begin{tabular}{|c|c|c|c|c|c|}
\hline \multicolumn{3}{|c|}{ AD patients } & \multicolumn{3}{c|}{ Control subjects } \\
\hline Identification & Age & MMSE & Identification & Age & MMSE \\
\hline Alz-1 & 69 & 24 & Con-1 & 68 & 30 \\
\hline Alz-2 & 71 & 15 & Con-2 & 61 & 29 \\
\hline Alz-3 & 67 & 12 & Con-3 & 70 & 30 \\
\hline Alz-4 & 56 & 14 & Con-4 & 64 & 30 \\
\hline Alz-5 & 79 & 9 & Con-5 & 60 & 30 \\
\hline Alz-6 & 64 & 15 & Con-6 & 63 & 30 \\
\hline Alz-7 & 59 & 20 & Con-7 & 73 & 29 \\
\hline Alz-8 & 60 & 16 & Con-8 & 69 & 29 \\
\hline Alz-9 & 72 & 15 & Con-9 & 56 & 27 \\
\hline Alz-10 & 71 & 15 & Con-10 & 74 & 30 \\
\hline Mean & 66.3 & 15.5 & Mean & 65.8 & 29.4 \\
\pm SD & \pm 7.1 & \pm 4.1 & $\pm S D$ & \pm 5.9 & \pm 1.0 \\
\hline
\end{tabular}

\section{B. $M E G$ recording}

MEGs were recorded using a 148-channel whole-head magnetometer (MAGNES $2500 \mathrm{WH}, 4 \mathrm{D}$ Neuroimaging) in a magnetically shielded room. While lying comfortably on a patient bed, subjects were asked to stay awake and to avoid head and eye movements. With the subjects in a relaxed state and eyes closed, 5 minutes of recording (sampling frequency of $678.17 \mathrm{~Hz}$ ) were acquired. These recordings were downsampled to $169.549 \mathrm{~Hz}$ (50863 data points).

All data were digitally filtered using a band-pass filter with cut-off frequencies at $0.4 \mathrm{~Hz}$ and at $70 \mathrm{~Hz}$ in order to reject artifacts. Low-frequency artifacts were typically due to eye movements and high-frequency ones were typically due to muscle activity. A second-order notch filter at $50 \mathrm{~Hz}$ was used to remove the line frequency.

Artifact-free epochs of 3392 samples (20 seconds) were selected from the MEG signal. The epochs were copied as ASCII files to a personal computer for further off-line analysis.

\section{Lempel-Ziv complexity}

The Lempel-Ziv (LZ) complexity for sequences of finite length was suggested by Lempel and Ziv [15]. It is a nonparametric, simple-to-calculate measure of complexity in a onedimensional signal that does not require long data segments to compute [20]. LZ complexity is related to the number of distinct substrings and the rate of their recurrence along the given sequence [21], with larger values corresponding to more complexity in the data.

LZ complexity analysis is based on a coarse-graining of the measurements, so before calculating the complexity measure $c(n)$, the signal must be transformed into a finite symbol sequence.
The simplest way is selected to convert time series values $[x(i), i=1,2, \ldots, N]$ into a sequence of characters (zero and one). The median value is estimated as a threshold $T_{d}$, as partitioning about the median is robust to outliers [25]. By comparison with the threshold, the signal data are converted into a $0-1$ sequence $P=s(1), s(2), \ldots, s(n)$, with $s(i)$ defined by [22]:

$$
s(i)= \begin{cases}0 & \text { if } x(i)<T_{d} \\ 1 & \text { if } x(i) \geq T_{d}\end{cases}
$$

The sequence $P$ is scanned from left to right increasing the complexity counter $c(n)$ by one unit every time a new subsequence of consecutive characters is encountered. The complexity measure can be estimated using the following algorithm [20] [22] [23]:

1) Let $S$ and $Q$ denote two subsequences of $P$ and $S Q$ be the concatenation of $S$ and $Q$, while sequence $S Q \pi$ is derived from $S Q$ after its last character is deleted ( $\pi$ means the operation to delete the last character in the sequence). Let $v(S Q \pi)$ denote the vocabulary of all different subsequences of $S Q \pi$. At the beginning, $c(n)=1, S=s(1), Q=s(2)$, therefore, $S Q \pi=s(1)$.

2) In general, $S=s(1), s(2), \ldots, s(r), Q=s(r+1)$, then $S Q \pi=s(1), s(2), \ldots, s(r)$; if $Q$ belongs to $v(S Q \pi)$, then $Q$ is a subsequence of $S Q \pi$, not a new sequence.

3) Renew $Q$ to be $s(r+1), s(r+2)$ and judge if $Q$ belongs to $v(S Q \pi)$ or not.

4) Repeat the previous steps until $Q$ does not belong to $v(S Q \pi)$. Now $Q=s(r+1), s(r+2), \ldots, s(r+i)$ is not a subsequence of $S Q \pi=s(1), s(2), \ldots, s(r+i-1)$, so increase $c(n)$ by one.

5) Thereafter, $S$ is renewed to be $S=$ $s(1), s(2), \ldots, s(r+i)$, and $Q=s(r+i+1)$.

These procedures have to be repeated until $Q$ is the last character. At this time the number of different subsequences in $P$-the measure of complexity- is $c(n)$.

In order to obtain a complexity measure which is independent of the sequence length, $c(n)$ should be normalized. If the length of the sequence is $n$ and the number of different symbols in the symbol set is $\alpha$, it has been proved [15] that the upper bound of $c(n)$ is given by:

$$
c(n)<\frac{n}{\left(1-\varepsilon_{n}\right) \log _{\alpha}(n)}
$$

where $\varepsilon_{n}$ is a small quantity and $\varepsilon_{n} \rightarrow 0(n \rightarrow \infty)$. In general, $n / \log _{\alpha}(n)$ is the upper bound of $c(n)$, where the base of the logarithm is $\alpha$, i.e.,

$$
\lim _{n \rightarrow \infty} c(n)=b(n) \equiv \frac{n}{\log _{\alpha}(n)}
$$

For a $0-1$ sequence, $\alpha=2$, therefore

$$
b(n) \equiv \frac{n}{\log _{2}(n)}
$$

and $c(n)$ can be normalized via $b(n)$ :

$$
C(n)=\frac{c(n)}{b(n)}
$$


$C(n)$, the normalized LZ complexity, reflects the arising rate of new patterns along with the sequence. Thus, it captures the temporal structure of the sequence.

\section{Statistical analysis}

The analyses were carried out separately for each channel. In order to determine if there are any differences between the LZ complexity values in both groups, control subjects and AD patients, a Student $t$-test was used.

\section{RESULTS}

We have used the LZ complexity to quantify the complexity in the time-series data. The epoch length was fixed at 3392 samples and we have coded the series about the median by a binary sequence (0-1) in the calculation of LZ complexity.

Fig. 1 resumes the average LZ complexity values estimated for the patients with $\mathrm{AD}$ and the control subjects, for the MEG channels (A1-A148). This average value is higher in the control group's MEG for all channels. Mean values were $0.78 \pm 0.06$ in the control group and $0.69 \pm 0.08$ in the $\mathrm{AD}$ patients group.

The LZ complexity values suggest that the complexity (in the sense of number of new subsequences in the data) in AD patients' MEGs is lower. This difference is statistically significant when using a Student $t$-test in 77 channels $(p<$ 0.01), as can be seen in Fig. 2. Fourteen of these channels (dark grey colored) have a $p<0.001$ (A16, A17, A32, A33, A34, A51, A52, A53, A69, A73, A74, A75, A93 and A112).

These results suggest that MEG activity of AD patients is less complex in certain regions than in a normal brain.

\section{DISCUSSION AND CONCLUSION}

MEG is a non-invasive neurophysiological technique that allows measuring the magnetic fields generated by brain activity, with millisecond temporal resolution and spatial resolution exceeding that of conventional EEG [26]. Although it was firstly used more than 30 years ago, MEG is still widely considered a new neurophysiological technique. To our knowledge the present study is the first that has used the LZ complexity to study multichannel MEG data in AD patients.

LZ complexity is an easy and fast method to measure the time series complexity. Only two simple mathematical operations are needed for the calculation of the LZ complexity: sequence comparison and number accumulation. It is a coarse-graining measure because the MEG data are transformed into a pattern whose elements are only two symbols (0-1). Although other conversions with more symbols could keep more information, previous studies have demonstrated that a binary conversion is enough to study the complexity of a system [22]. Moreover, the median value is robust to outliers. These advantages support the proposal that the LZ complexity is a good measure of the complexity of biological signals as the MEG.

In this research $\mathrm{AD}$ patients group and control group were carefully matched for age (AD patients, mean age \pm SD 66.3 \pm 7.1 years; control subjects, mean age \pm SD $65.8 \pm 5.9$ years). Therefore, the complexity loss may well represent the cognitive dysfunction in $\mathrm{AD}$. It is remarkable that the accuracy in the clinical diagnosis of AD is less than $90 \%$. So, it is possible that not all the patients have AD.

In summary, our results suggest that the LZ complexity is a good method to differentiate between the two groups we

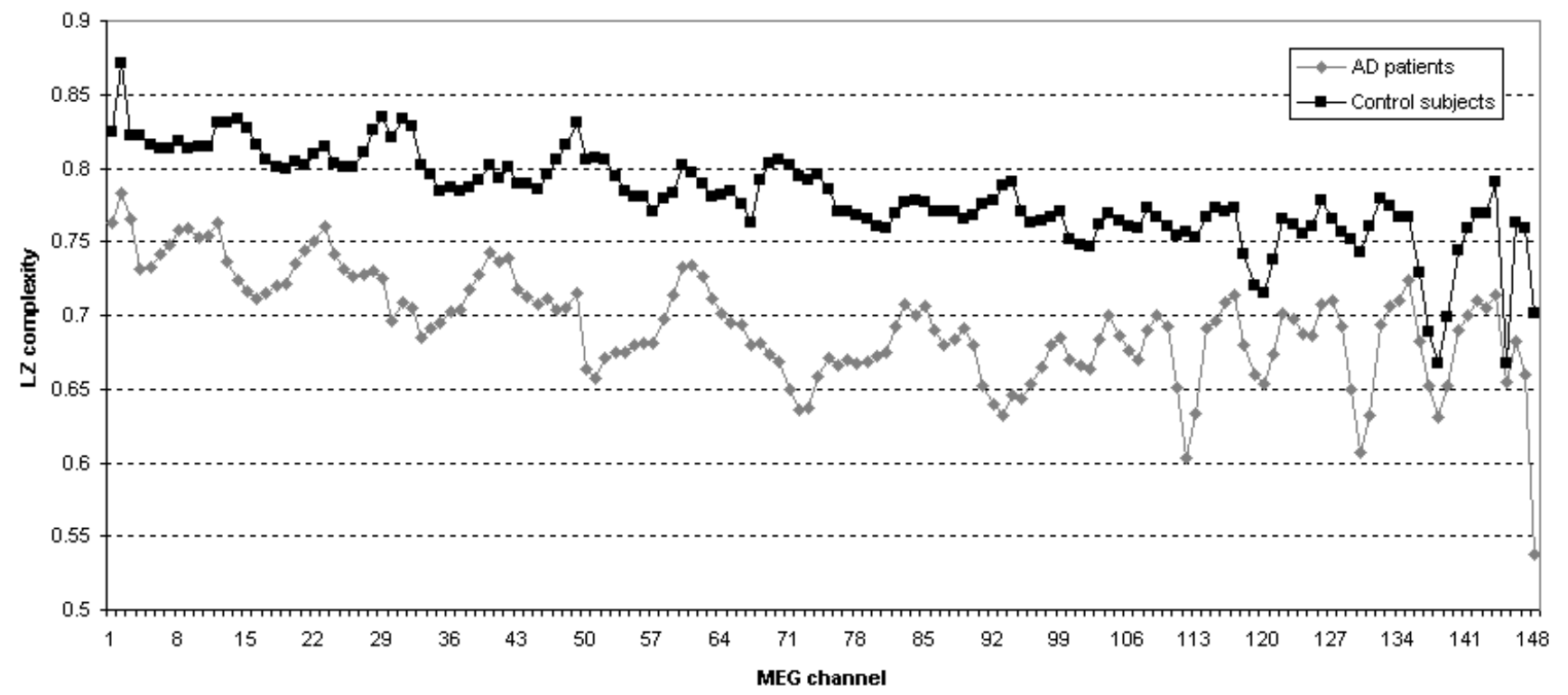

Fig. 1. The average LZ complexity values of the MEGs in Alzheimer disease patients and control subjects for all channels, from A1 to A148. 


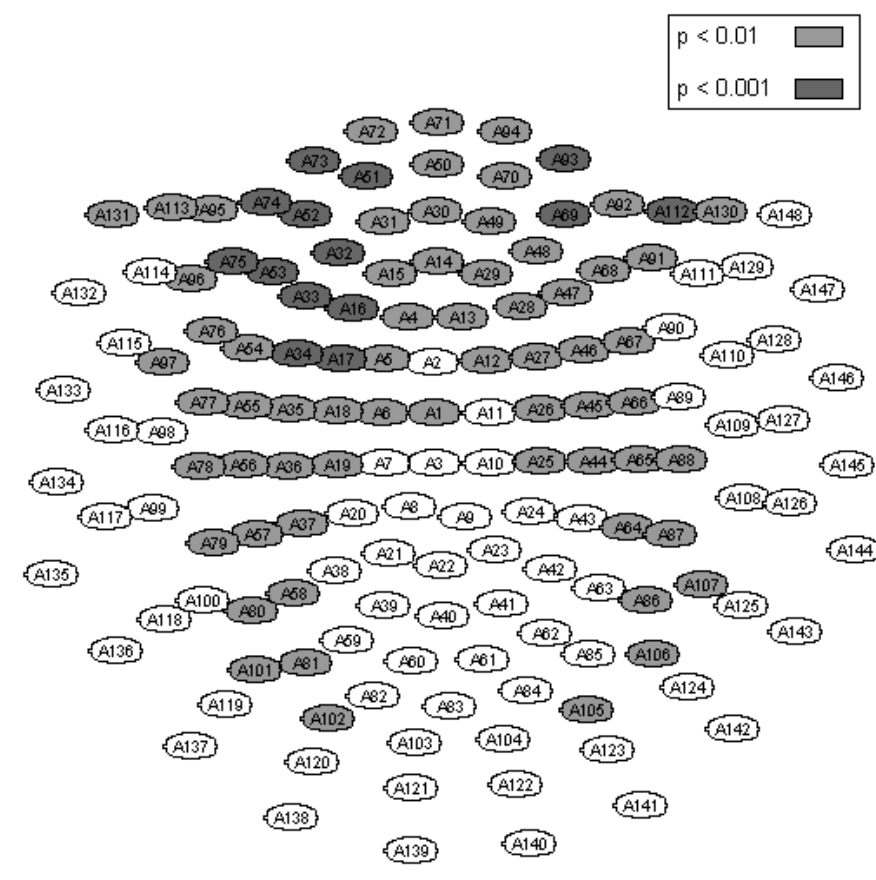

Fig. 2. Squids diagram with $\mathrm{p}$ values.

have studied. Nevertheless, more AD patients and control subjects' recordings are needed to confirm the preliminary results obtained in this research.

\section{ACKNOWLEDGMENT}

This research has been supported by project from the Consejería de Fomento de la Junta de Castilla y León. The authors are very grateful to María Rosario Peña, who kindly revised the English version of this paper.

\section{REFERENCES}

[1] J. Jeong, "EEG dynamics in patients with Alzheimer's disease," Clin. Neurophysiol., vol. 115, pp. 1490-1505, 2004.

[2] R. C. Petersen, Ed., Mild cognitive impairment: aging to Alzheimer's Disease. New York: Oxford University Press, 2003.

[3] T. D. Bird, "Harrison's principles of internal medicine." in Alzheimer's disease and other primary dementias, E. Braunwald, A. S. Fauci, D. L. Kasper, S. L. Hauser, D. L. Longo, and J. L. Jameson, Eds. New York: The McGraw-Hill Companies Inc, 2001, pp. 2391-2399.

[4] B. J. Cummings, C. J. Pike, R. Shankle, and C. W. Cotman, "Betaamyloid deposition and other measures of neuropathology predict cognitive status in Alzheimer's disease." Neurobiol. Aging, vol. 17, pp. 921-933, 1996.

[5] J. Q. Trojanowski, "The cellular and molecular correlates of cognitive impairments in the Alzheimer's disease brain." Neurobiol. Aging, vol. 17, pp. 941-943, 1996.

[6] M. F. Folstein, S. E. Folstein, and P. R. McHugh, "Mini-mental state. a practical method for grading the cognitive state of patients for the clinician," J. Psychiatr. Res., vol. 12, pp. 189-198, 1975.
[7] C. J. Stam, T. Montez, B. F. Jones, S. A. R. B. Rombouts, Y. van der Made, Y. A. L. Pijnenburg, and P. Scheltens, "Disturbed fluctuations of resting state EEG synchronization in Alzheimer's disease," Clin. Neurophysiol., vol. 116, pp. 708-715, 2005.

[8] J. Jeong, S. Y. Kim, and S.-H. Han, "Non-linear dynamical analysis of the EEG in Alzheimer's disease with optimal embedding dimension," Electroen. Clin. Neuro., vol. 106, pp. 220-228, 1998.

[9] B. Jelles, J. van Birgelen, J. Slaets, R. Hekster, E. Jonkman, and C. Stam, "Decrease of non-linear structure in the EEG of Alzheimer patients compared to healthy controls," Clin. Neurophysiol., vol. 110, pp. 1159-1167, 1999.

[10] P. Grassberger and I. Procaccia, "Characterization of strange attractors," Pyis. Rev. Lett., vol. 50, no. 5, pp. 346-349, Jan. 1983.

[11] J. P. Eckmann and D. Ruelle, "Fundamental limitations for estimating dimensions and Lyapunov exponents in dynamical systems," Physica $D$, vol. 56, pp. 185-187, 1992.

[12] A.-M. van Cappellen van Walsum, Y. Pijnenburg, H. Berendse, B. van Dijk, D. Knol, P. Scheltens, and C. Stam, "A neural complexity measure applied to MEG data in Alzheimer's disease," Clin. Neurophysiol., vol. 114, pp. 1034-1040, 2003.

[13] J. Jeong, J. C. Gore, and B. S. Peterson, "Mutual information analysis of the EEG in patients with Alzheimer's disease," Clin. Neurophysiol., vol. 112, pp. 827-835, 2001.

[14] T. Koenig, L. Prichep, T. Dierks, D. Hubl, L. O. Wahlund, E. R. John, and V. Jelic, "Decreased EEG synchronization in Alzheimer's disease and mild cognitive impairment," Neurobiol. Aging, vol. 26, pp. 165171, 2005.

[15] A. Lempel and J. Ziv, "On the complexity of finite sequences," IEEE Trans. Inform. Theory, vol. 22, pp. 75-81, 1976.

[16] L. Huang, P. Yu, F. Ju, and J. Cheng, "Prediction of response to incision using the mutual information of electroencephalograms during anaesthesia," Med. Eng. Phys., vol. 25, pp. 321-327, 2003.

[17] J. W. Zhang, C. X. Zheng, and D. Z. Jiang, "EEG complexity measurement of focal ischemic cerebral injury," in Proceedings of the 20th Annual Conference of the IEEE Engineering in Medicine and Biology Society, vol. 20, no. 4, 1998, pp. 2027-2029.

[18] X. Wu and J. Xu, "Complexity and brain functions," Acta Biophysica Sinica, vol. 7, pp. 103-106, 1991.

[19] J. Xu, Z. rong Liu, R. Liu, and Q. fei Yang, "Information transmission in human cerebral cortex," Physica D, vol. 106, pp. 363-374, 1997.

[20] X. S. Zhang, Y. S. Zhu, N. V. Thakor, and Z. Z. Wang, "Detecting ventricular tachycardia and fibrillation by complexity measure," IEEE Trans. Biomed. Eng., vol. 46, pp. 548-555, May 1999.

[21] N. Radhakrishnan and B. N. Gangadhar, "Estimating regularity in epileptic seizure time-series data," IEEE Eng. Med. Biol. Mag., vol. 17, no. 3, pp. 89-94, 1998.

[22] X. S. Zhang, R. J. Roy, and E. W. Jensen, "EEG complexity as a measure of depth of anesthesia for patients," IEEE Trans. Bio-Med. Eng., vol. 48, no. 12, pp. 1424-1433, Dec. 2001.

[23] X. S. Zhang and R. J. Roy, "Derived fuzzy knowledge model for estimating the depth of anesthesia," IEEE Trans. Bio-Med. Eng., vol. 48, no. 3, pp. 312-323, Mar. 2001.

[24] G. McKhann, D. Drachman, and M. Folstein, "Clinical diagnosis of Alzheimer's disease: report of NINCDS-ADRDA work group under the auspices of department of health and human services task force on Alzheimer's disease," Neurology, vol. 34, pp. 939-944, 1984.

[25] R. Nagarajan, "Quantifying physiological data with Lempel-Ziv complexity-certain issues," IEEE Trans. Bio-Med. Eng., vol. 49, no. 11, pp. 1371-1373, Nov. 2002.

[26] D. Osipova, J. Ahveninen, S. Kaakkola, I. P. Jaaskelainen, J. Huttunen, and E. Pekkonen, "Effects of scopolamine on MEG spectral power and coherence in elderly subjects," Clin. Neurophysiol., vol. 114, pp. 19021907, 2003. 